Objective To assess the current state of knowledge and practice regarding SRC (diagnosis, treatment, return to play) within the French-speaking sports community in order to tailor the prevention approaches.

Design Multicentric cross sectional survey.

Setting An online survey ( 15 minutes completion time) was sent through mailing lists and social networks. The survey was available for three months and monthly reminders were sent.

Patients (or Participants) Athletes, sports healthcare professionals, and coaches through the ReFORM network

Interventions (or Assessment of Risk Factors) N/A

Main Outcome Measurements Reported level of knowledge regarding SRC and access to educational resources.

Results 2072 participants took part in the survey and 1704 finished it (completion rate: $82 \%$ ). The sample included $48 \%$ of athletes, $33 \%$ of coaches and 19\% of healthcare professionals. The main countries represented were France (35\%), Canada (32\%) and Belgium (12\%). The preliminary analyses reported a SRC knowledge self-assessment as 'good' or 'excellent' in $87 \%$ of healthcare professionals and $69 \%$ of coaches; while more than $40 \%$ of athletes rated their knowledge as 'poor' or 'none'. Only 17\% of athletes reported knowing about a SRC education programme in their setting against $63 \%$ for healthcare professionals and $45 \%$ for coaches. Regarding coaches, $54 \%$ do not feel having sufficient professional resources to correctly manage a SRC over the return to sports continuum.

Conclusions There seems to be a great interest from field stakeholders reflected by the completion rate. These preliminary results show a discrepancy in the level of SRC knowledge and the access to educational resources between athletes, coaches and healthcare professionals.

\section{DO CERVICAL SPINE, VESTIBULO-OCULAR, DYNAMIC BALANCE, AND DIVIDED ATTENTION MEASURES IN ELITE YOUTH ICE HOCKEY PLAYERS RETURN TO BASELINE LEVELS AT TIME OF MEDICAL CLEARANCE TO RETURN TO PLAY?}

1,2,3,4,5 Kathryn Schneider, ${ }^{5,6}$ Geoff Schneider, ${ }^{1,2,3,7}$ Carolyn Emery. ${ }^{~}$ Sport Injury Prevention Research Centre, Faculty of Kinesiology, University of Calgary, Calgary, Canada; ${ }^{2}$ Alberta Children's Hospital Research Institute, for Child and Maternal Health, Faculty of Medicine, University of Calgary, Calgary, Canada; ${ }^{3}$ Hotchkiss Brain Institute, University of Calgary, Calgary, Canada; ${ }^{4}$ Sport Medicine Centre, University of Calgary, Calgary, Canada; ${ }^{5}$ Evidence Sport and Spinal Therapy, Calgary, Canada; ${ }^{6}$ Department of Radiology, Cummings School of Medicine, University of Calgary, Calgary, Canada; 'Departments of Pediatrics and Community Health Sciences, Cumming School of Medicine, University of Calgary, Calgary, Canada

\subsection{6/bjsports-2021-IOC. 162}

Background Evaluation of multiple sensory and motor domains at time of return to play (RTP) may inform risk of recurrent concussion and injury following concussion.

Objective To evaluate if measures of (1) cervical spine function, (2) vestibulo-ocular reflex (VOR) function, (3) dynamic balance and (4) tasks of divided attention have returned to preinjury levels at medical clearance to RTP in elite youth ice hockey players.

Design Case series nested in a prospective cohort study $(\mathrm{n}=559)$.

Setting Canadian youth ice hockey.

Participants Youth ice hockey players [13-17 years; $n=45$ (8 female, 37 male)].
Interventions Players who were diagnosed with an ice hockey-related concussion completed preseason and RTP measures.

Main Outcome Measurements Cervical spine measures (cervical flexor endurance test, head perturbation test, anterolateral strength, cervical flexion rotation test, joint position error), VOR tests [head thrust test, dynamic visual acuity (clinical and computerized)], dynamic balance tests (functional gait) and divided attention tasks (walking-while-talking-test WWTT) were included. Non-parametric (Wicoxon signed-rank, Stuart-Maxwell) analyses compared preseason to RTP scores.

Results Symptoms of dizziness, neck pain, and headache were reported by 29\%, 18\% and 20\% fewer players at RTP than preseason respectively. Anterolateral cervical muscle strength $(\mathrm{z}=-5.16, \mathrm{p}<0.0001)$ and joint position error (left) $(\mathrm{z}=2.91$, $\mathrm{p}=0.0036)$ were poorer at RTP compared to preseason. The WWTT time $(\mathrm{z}=-2.66, \mathrm{p}=0.0079)$ and FGA scores were improved at RTP $(\mathrm{z}=-2.55, \mathrm{p}=0.011)$.

Conclusions Anterolateral cervical spine strength and joint position error (left) did not return to preseason values at RTP and may suggest incomplete recovery not indicated by symptoms at RTP. WWTT and dynamic balance were improved at RTP. Further evaluation of clinical outcomes on risk of subsequent injury following concussion is warranted.

\section{PRIMARY PREVENTION OF SPORT-RELATED CONCUSSION IN YOUTH ICE HOCKEY: A PILOT RANDOMIZED CONTROLLED TRIAL}

\begin{abstract}
1,2,3,4,5Kathryn Schneider, ${ }^{1,2,3}$ Paul Eliason, ${ }^{1}$ Cody van Rassel, ${ }^{1,4}$ Stacy Sick, ${ }^{1}$ Lauren Miurz, ${ }^{5,6} \mathrm{Geoff}$ Schneider, ${ }^{1,2,3}$ Carolyn Emery. ${ }^{1}$ Sport Injury Prevention Research Centre, Faculty of Kinesiology, University of Calgary, Calgary, Canada; ${ }^{2}$ Alberta Children's Hospital Research Institute, Calgary, Canada; ${ }^{3}$ Hotchkiss Brain Institute, University of Calgary, Calgary, Canada; ${ }^{4}$ Acute Sport Concussion Clinic, Sport Medicine Centre, Faculty of Kinesiology, University of Calgary, Calgary, Canada; ${ }^{5}$ Evidence Sport and Spinal Therapy, Calgary, Canada; ${ }^{6}$ Department of Radiology, Cumming School of Medicine, University of Calgary, Calgary, Canada
\end{abstract}

\subsection{6/bjsports-2021-IOC.163}

Background Neuromuscular and sensorimotor training may reduce the risk of concussion.

Objective To evaluate the feasibility and efficacy of a concussion prevention neuromuscular training program in youth ice hockey.

Design Pilot cluster-randomized controlled trial.

Setting Canadian ice hockey.

Patients (or Participants) Youth ice hockey players (ages 1317).

Interventions (or Assessment of Risk Factors) A study physiotherapist visited teams weekly for six weeks. Both study groups received standard concussion education and previously validated concussion surveillance. Intervention teams completed a progressive neuromuscular and sensorimotor training program (10-20 minutes per session) (including aerobic, balance, strength, agility, adaptation, cervical spine strength and dividing attention), on- and off-ice. Control teams completed their typical warm-up.

Main Outcome Measurements Recruitment, completion, retention rates, time and safety were used to evaluate feasibility. Sport-related concussion was defined using the $5^{\text {th }}$ Consensus Statement on Concussion in Sport. 
Results Eight teams [ $\mathrm{n}=118$ players; 83 males, 35 females] consented to participate. All control teams and $3 / 4$ of the intervention teams completed all 6 sessions [median 85 days (range 42-102)]. No adverse events were reported. There were 6 concussions in the intervention group $(n=65)$ and 4 in the control group $(n=53)$. Exploratory univariate Poisson regression analysis adjusted for cluster by team revealed no difference in concussion risk between groups [incidence rate ratio $(\mathrm{IRR})=0.99$ concussions $/ 100$ players/season $(95 \% \mathrm{CI} ; 0.28-3.48)$. For males alone, there was also no difference in IRR $\left[\mathrm{IRR}_{\text {males }}=0.37(95 \% \mathrm{CI}\right.$; 0.068-1.94)]. Both female teams were allocated to the intervention group.

Conclusions The implementation of a neuromuscular and sensorimotor training program with youth ice hockey teams was feasible and safe. Future evaluation in a larger sample over a longer training period to examine the effects of neuromuscular and sensorimotor training strategies on the risk of concussion in youth ice hockey players, including consideration of sex and age group is warranted.

\section{THE EFFECT OF THE FÉDÉRATION INTERNATIONALE DE FOOTBALL ASSOCIATION (FIFA) 11+ KIDS INJURY PREVENTION PROGRAM ON REDUCING INJURY RATES IN CHILDREN'S SOCCER: A CLUSTER-RANDOMIZED CONTROLLED TRIAL}

\begin{abstract}
1,2,3 Wesam Saleh A Al Attar, ${ }^{4}$ Saud Alarifi, ${ }^{5}$ Hussain Saleh H Ghulam, ${ }^{6}$ Hosam Alzahrani, ${ }^{7}$ Msaad M Alzhrani, ${ }^{3}$ Ross H Sanders. 'Department of Physical Therapy, Faculty of Applied Medical Science, Umm Al Qura University, Makkah, Saudi Arabia; ${ }^{2}$ Department of Sport, Exercise and Health, Faculty of Medicine, University of Basel, Basel, Switzerland: ${ }^{3}$ Discipline of Exercise and Sport Science, Faculty of Medicine and Health, The University of Sydney, Sydney, Australia; ${ }^{4}$ Department of Physical Therapy, King Abdulaziz Medical City, Riyadh, Saudi Arabia; ${ }^{5}$ Department of Rehabilitation Medical Sciences, Faculty of Applied Medical Science, Najran University, Najran, Saudi Arabia; ${ }^{6}$ Department of Physiotherapy, College of Applied Medical Sciences, Taif University, Taif, Saudi Arabia; ${ }^{7}$ Department of Physical Therapy, Faculty of Applied Medical Science, Majmaah University, Majmaah, Saudi Arabia
\end{abstract}

\subsection{6/bjsports-2021-IOC.164}

Background The Fédération International de Football Association (FIFA) $11+$ Kids injury prevention program is an exercise-based program developed by an international group of experts to prevent injuries among children's soccer players.

Objective The objective of this study is to evaluate the effect of the FIFA $11+$ Kids injury prevention program on reducing the incidence of injuries among children soccer players aged 7-13 years.

Design A cluster-randomized controlled trial.

Setting Children's soccer.

Patients (or Participants) Ninety-four boys' soccer teams (780) players (under 8 years, under 9 years, under 11 years, and under 13 years age groups) were randomly allocated into the experimental or a control group.

Interventions (or Assessment of Risk Factors) The experimental group performed the FIFA $11+$ Kids injury prevention program as warm-up during training sessions for at least twice a week, and the control group performed their usual warm-ups. Participants were prospectively followed during one season.

Main Outcome Measurements The primary outcome was to investigate the incidence of initial, recurrent injury, mechanism of injury and injury severity.

Results A total of 43 injuries were reported among 391 players in the experimental group in 8353.33 hours of exposure
(0.85 injuries/1000 exposure hours), and a total of 86 injuries were reported among 389 players in the control group in 7102.67 hours of exposure (2.01 injuries/1000 exposure hours). The injury risk ratio IRR was 0.43 , which suggests that the injuries in the experimental group were $57 \%$ less in comparison to the control group.

Conclusions Implementation of the FIFA $11+$ Kids injury prevention program reduced overall injury rates in boys' soccer players more than the usual warm-up.

\section{THE THE FÉDÉRATION INTERNATIONALE DE FOOTBALL ASSOCIATION (FIFA) 11+ KIDS INJURY PREVENTION PROGRAM: AWARENESS, IMPLEMENTATION, AND OPINION OF WORLDWIDE CHILDREN'S SOCCER COACHES}

1,2,3 Wesam Saleh A Al Attar, 'Saad M Alhosaini, 'Sami Z Alharbi, 'Yasser M Alraddadi, ${ }^{4}$ Hussain Saleh H Ghulam, ${ }^{5}$ Mashaer Alyami, ${ }^{3}$ Ross H Sanders. 'Department of Physical Therapy, Faculty of Applied Medical Science, Umm Al Qura University, Makkah, Saudi Arabia; ${ }^{2}$ Department of Sport, Exercise and Health, Faculty of Medicine, University of Basel, Basel, Switzerland; ${ }^{3}$ Discipline of Exercise and Sport Science, Faculty of Medicine and Health, The University of Sydney, Sydney, Australia; ${ }^{4}$ Department of Rehabilitation Medical Sciences, Faculty of Applied Medical Science, Najran University, Najran, Saudi Arabia; ${ }^{5}$ King Fahad Specialist Hospital, Dammam, Saudi Arabia

\subsection{6/bjsports-2021-IOC.165}

Background The Fédération Internationale de Football Association (FIFA) has promoted the FIFA $11+$ injury prevention program worldwide. The FIFA $11+$ injury prevention program for kids was developed and shown to be effective in reducing soccer-related injuries in numerous studies.

Objective To assess the awareness, implementation, and opinion of worldwide children's soccer coaches regarding the FIFA $11+$ Kids injury prevention program.

Design Cross-sectional study.

Setting An online survey for all continental football federations. Patients (or Participants) A total of 486 children's soccer coaches completed the survey.

Interventions (or Assessment of Risk Factors) The questionnaire consisted of questions relating to awareness, implementation, and coaches' opinions of the FIFA $11+$ Kids injury prevention program.

Main Outcome Measurements The awareness level, implementation rate, and opinion of the the FIFA $11+$ Kids injury prevention program efficacy.

Results A total of 202 (41.6\%) of the children's soccer coaches reported awareness of the FIFA $11+$ Kids injury prevention program and 155 (31.9\%) were reported implementing the FIFA $11+$ Kids injury prevention program in their current practice. Participants who reported implementation of the FIFA $11+$ Kids injury prevention program also reported a positive attitude towards the program efficacy, with a score of $8.19 \pm 0.88$ out of 10 .

Conclusions Globally, children's soccer coaches showed poor awareness levels of the FIFA $11+$ Kids injury prevention program, however, the implementation level among coaches who reported awareness was relatively high. A high number of children's soccer coaches reported a positive perception regarding the efficacy of the FIFA $11+$ Kids injury prevention program in reducing and preventing injuries. Therefore, there is a need for further efforts and research to increase the international awareness and implementation rate of the FIFA $11+$ Kids injury prevention program. 